\title{
Robustness of homogeneous and locally homogeneous differential inclusions
}

\author{
Emmanuel Bernuau, Denis Efimov, Wilfrid Perruquetti
}

\begin{abstract}
The paper proposes constructive conditions for verification of input-to-state stability property for discontinuous systems using geometric homogeneity. Two sets of conditions are developed: for a class of homogeneous and locally homogeneous systems described by differential inclusions. The advantage of the proposed conditions is that they are not based on the Lyapunov function method, but more related to algebraic operations over the right-hand side of the system.
\end{abstract}

\section{INTRODUCTION}

The problem of robustness and stability analysis with respect to external inputs (like exogenous disturbances or measurement noises) for dynamical systems is in the center of attention of many research works [1], [2], [3], [4], [5], [6]. One of the most popular theories, which can be used for this robustness analysis of nonlinear systems, was originated more than 20 years ago [7] and it is based on the Input-toState Stability (ISS) property and many related notions. The advantages of ISS theory include a complete list of necessary and sufficient conditions, existence of the Lyapunov method extension, a rich variety of stability concepts adopted for different control and estimation problems.

The main tool to check the ISS property for a nonlinear system consists in a Lyapunov function design satisfying sufficient conditions. As usual, there is no generic approach to select a Lyapunov function for nonlinear systems. Therefore, computationally tractable approaches for ISS verification for particular classes of nonlinear systems are of great importance, and they are highly demanded in applications. In this work we are going to propose and extend such techniques for checking ISS and Input-to-State Practical Stability (ISpS) for a class of homogeneous and locally homogeneous discontinuous systems.

The homogeneity is an intrinsic property of an object on which the flow of a particular vector field, called Euler

This work was supported by EU Interreg IV A 2 Mers Seas Zeeën Crossborder Cooperation Programme under SYSIASS project 06-020. It was also partly supported by Ministry of Higher Education and Research, Nord-Pas de Calais Regional Council and FEDER through the "Contrat de Projets Etat Region (CPER) CIA 2007-2013", by the Government of Russian Federation (Grant 074-U01) and the Ministry of Education and Science of Russian Federation (Project 14.Z50.31.0031). This work was also supported by ANR Grant CHASLIM (ANR-11-BS03-0007).

E. Bernuau is with Research Center "E. Piaggio", Faculty of Engineering, University of Pisa, Largo Lucio Lazzarino 1, 56122 Pisa, Italy; emmanuel.bernuau@gmail.com.

D. Efimov and W. Perruquetti are with Non-A team of INRIA - LNE, Parc Scientifique de la Haute Borne, 40 avenue Halley, Bat.A, Park Plaza, 59650 Villeneuve d'Ascq, France and with LAGIS UMR CNRS 8219, Ecole Centrale de Lille, BP 48, Cité Scientifique, 59651 Villeneuve-d'Ascq, France; denis.efimov@inria.fr, wilfrid.perruquetti@inria.fr. D. Efimov is with the Department of Control Systems and Informatics, National Research University ITMO, 49 avenue Kronverkskiy, 197101 Saint Petersburg, Russia. vector field, operates as a scaling. This property entails a lot of qualitative results for a homogeneous object, and is of particular interest in view of stability purposes. The notion of homogeneity was found useful by many authors [8], [9], [10], [11], [12], [13], [14], [15]. The main feature of this property is that any local property of the system is in fact global. Obviously, some systems are not homogeneous. The local homogeneity notion has been proposed in [8], [16], [17], it allows a local approximation of a system by a homogeneous one to be obtained. Qualitative properties of the homogeneous approximation are shown to persist locally for the starting system.

The ISS properties of homogeneous or locally homogeneous continuous systems have been studied in [18], [19], [16], [20]. But continuity assumption is not always verified. For instance, mechanical systems with friction or systems controlled by a Sliding Mode Control (SMC) induce a discontinuous vector field. In this work, ISS and ISpS properties for discontinuous systems and systems which dynamics are given by a Differential Inclusion (DI) are provided.

Numerous frameworks have been given to deal with discontinuous systems. We will focus here on the Filippov's solution [21]. Filippov's idea is to replace a (discontinuous) vector field by a set-valued map, mapping a point to a set of admissible velocities. The solutions are then absolute continuous curves which derivative belongs to this set of admissible velocities, leading hence to a DI. Different notions of homogeneity for DI have been proposed [21], [22], [23], [24]. In the last paper, a converse homogeneous Lyapunov theorem was proved, on which we shall rely to prove ISS properties. This result was already used to get ISS properties for DI in [25].

In this work, our objective is twofold. First, we shall generalize the notion of local homogeneity to differential inclusions and second we shall formulate conditions of ISS and ISpS properties of discontinuous systems using homogeneity and local homogeneity. We will present these results using geometric homogeneity to have the most generic formulation.

The outline of the paper is as follows. Section II is devoted to the introduction of notations and results that will be used in the sequel. Section III presents the new framework of homogenization of a DI and the associated stability results. Section IV gives the ISS and ISpS results obtained using homogeneity techniques. Finally, a conclusion will sum up the paper and will give some perspectives. 


\section{Preliminaries}

\section{A. Notations}

We denote $n$ a positive integer and we will be interested in systems defined on $\mathbb{R}^{n}$. We endow $\mathbb{R}^{n}$ with the Lebesgue measure and denote $\mathcal{N}$ the set of all zero-measure subsets of $\mathbb{R}^{n}$. For $x \in \mathbb{R}^{n}$ and $\varepsilon>0$, we denote $B(x, \varepsilon)$ the open ball centered in $x$ and of radius $\varepsilon$.

In this paper, locally essentially bounded vector fields are considered. The set of locally essentially bounded vector fields is denoted by $\mathcal{L}_{\text {loc }}^{\infty}\left(\mathbb{R}^{n}, \mathbb{R}^{n}\right)$.

Definition 2.1: The set of nonempty compact subsets of $\mathbb{R}^{n}$ is denoted by $\mathfrak{H}\left(\mathbb{R}^{n}\right)$. The Hausdorff distance between $X, Y \in \mathfrak{H}\left(\mathbb{R}^{n}\right)$ is defined by:

$$
\mathfrak{d}(X, Y)=\max \left(\sup _{x \in X} \mathrm{~d}(x, Y), \sup _{y \in Y} \mathrm{~d}(y, X)\right),
$$

where the distance between a point and a compact set is defined by

$$
\mathrm{d}(x, Y)=\inf _{y \in Y}\|x-y\| .
$$

Proposition 2.2: The Hausdorff distance defines a distance on $\mathfrak{H}\left(\mathbb{R}^{n}\right)$. Endowed with this distance, $\left(\mathfrak{H}\left(\mathbb{R}^{n}\right), \mathfrak{d}\right)$ is a complete metric space. Moreover, for all $\lambda \in \mathbb{R}$, $\mathfrak{d}(\lambda X, \lambda Y)=|\lambda| \mathfrak{d}(X, Y)$.

We will denote by $\partial A$ the boundary of a bounded set $A$ and $\|A\|=\sup _{a \in A}\|a\|$. If $A$ is compact, $\|A\|=\mathfrak{d}(A,\{0\})$.

Definition 2.3: Let $(E, \mathrm{~d})$ be a metric space, and let $u_{k}$ : $\mathbb{R}^{n} \rightarrow E$ be a sequence of mappings. We say that this sequence converges uniformly on compact sets to $u: \mathbb{R}^{n} \rightarrow$ $E$, denoted $u_{k} \underset{k \rightarrow+\infty}{\stackrel{C U C}{\rightarrow}} u$, iff for any compact set $K \subset \mathbb{R}^{n}$ and for all $\varepsilon>0$ there exists a $k_{0}>0$ such that for all $k>k_{0}, \sup _{x \in K} \mathrm{~d}\left(u_{n}(x), u(x)\right)<\varepsilon$.

\section{B. Differential inclusions}

We refer to [21] and [26] for the basic definitions and the technical material on set-valued maps and DI. In this section, we will only recall the definitions and results that will be used hereafter, without any proof.

The Filippov's regularization procedure consists in the construction of a set-valued map $F$ starting with a vector field $f \in \mathcal{L}_{\text {loc }}^{\infty}\left(\mathbb{R}^{n}, \mathbb{R}^{n}\right)$ :

$$
\mathcal{F}[f](x)=\bigcap_{\varepsilon>0} \bigcap_{\mathrm{N} \in \mathcal{N}} \overline{\operatorname{conv}}(f(B(x, \varepsilon) \backslash \mathrm{N})) .
$$

By construction, for all $x \in \mathbb{R}^{n}$, the set $\mathcal{F}[f](x)$ is compact and convex. Moreover, the set-valued map $\mathcal{F}[f]$ is upper semicontinuous.

In many applications, the DI is given by the set-valued map coming from the Filippov's procedure. We will therefore focus on set-valued map with the properties inherited by this procedure.

Definition 2.4: Let $F$ be a set-valued map. We say that $F$ verifies the standard assumptions (SA) if $F$ is upper semicontinuous and if for any $x \in \mathbb{R}^{n}, F(x)$ is a nonempty compact convex set.

\section{Homogeneity}

To introduce the notion of geometric homogeneity, the class of Euler vector fields has to be defined.

Definition 2.5: [27] A vector field $\nu \in \mathcal{C}^{1}\left(\mathbb{R}^{n}, \mathbb{R}^{n}\right)$ is said to be Euler if it is complete and if the origin is a GAS equilibrium of $-\nu$.

We will always write $\Phi$ the flow of $\nu$, that is $\Phi^{s}(x)$ is the current state at time $s$ of the trajectory of $\nu$ starting from $x$ at $s=0$. We also denote $d_{x} \Phi^{s}$ the differential of the diffeomorphism $\Phi^{s}$ at a fixed $s \in \mathbb{R}$, taken at $x \in \mathbb{R}^{n}$. We are now able to state the classical definitions of geometric homogeneity.

Definition 2.6: Let $\nu$ be an Euler vector field.

- A function $V: \mathbb{R}^{n} \rightarrow \mathbb{R}$ is $\nu$-homogeneous of degree $\kappa \in \mathbb{R}$ if:

$$
V\left(\Phi^{s}(x)\right)=e^{\kappa s} V(x) \quad \forall x \in \mathbb{R}^{n}, \forall s \in \mathbb{R} .
$$

- A vector field $f: \mathbb{R}^{n} \rightarrow \mathbb{R}^{n}$ is $\nu$-homogeneous of degree $\kappa \in \mathbb{R}$ if:

$$
f\left(\Phi^{s}(x)\right)=e^{\kappa s} d_{x} \Phi^{s} f(x) \quad \forall x \in \mathbb{R}^{n}, \forall s \in \mathbb{R} .
$$

The relation (3) can be recast under a more compact form $\mathcal{H}_{\kappa}^{s}(f)=f$, where the vector field $\mathcal{H}_{\kappa}^{s}(f)$ is defined by:

$$
\mathcal{H}_{\kappa}^{s}(f): x \mapsto e^{-\kappa s}\left(d_{x} \Phi^{s}\right)^{-1} f\left(\Phi^{s}(x)\right) .
$$

\section{Homogeneous differential inclusions}

In this subsection, we recall some definitions and results obtained in [24] that we will need in the sequel.

Definition 2.7: [24] Let $\nu$ be an Euler vector field. A setvalued map $F: \mathbb{R}^{n} \rightrightarrows \mathbb{R}^{n}$ is $\nu$-homogeneous of degree $\kappa \in \mathbb{R}$ if for all $s \in \mathbb{R}$ we have:

$$
\mathcal{H}_{\kappa}^{s}(F)=F,
$$

where we extend the operator $\mathcal{H}_{\kappa}^{s}$ defined in (4) by:

$$
\mathcal{H}_{\kappa}^{s}(F): x \mapsto e^{-\kappa s}\left(d_{x} \Phi^{s}\right)^{-1} F\left(\Phi^{s}(x)\right) .
$$

Proposition 2.8: Let $f \in \mathcal{L}_{\text {loc }}^{\infty}\left(\mathbb{R}^{n}, \mathbb{R}^{n}\right)$ be a vector field. Then for all $s \in \mathbb{R}$ and all $\kappa \in \mathbb{R}$ we have:

$$
\mathcal{H}_{\kappa}^{s}(\mathcal{F}[f])=\mathcal{F}\left[\mathcal{H}_{\kappa}^{s}(f)\right] .
$$

Proof: Since for all $\varepsilon>0$ there exist $\varepsilon_{-}>0$ and $\varepsilon_{+}>$ 0 such that $\Phi^{s}\left(B\left(x, \varepsilon_{-}\right)\right) \subset B\left(\Phi^{s}(x), \varepsilon\right) \subset \Phi^{s}\left(B\left(x, \varepsilon_{+}\right)\right)$ we have $\mathcal{F}[f]\left(\Phi^{s}(x)\right)=$

$$
\begin{aligned}
& \bigcap_{\varepsilon>0} \bigcap_{N \in \mathcal{N}} \overline{\overline{\operatorname{conv}}}\left(f(y), y \in B\left(\Phi^{s}(x), \varepsilon\right) \backslash N\right) \\
= & \bigcap_{\varepsilon>0} \bigcap_{N \in \mathcal{N}} \overline{\overline{c o n v}}\left(f(y), y \in \Phi^{s}(B(x, \varepsilon)) \backslash N\right) \\
= & \bigcap_{\varepsilon>0} \bigcap_{N \in \mathcal{N}} \overline{\operatorname{conv}}\left(f\left(\Phi^{s}(z)\right), z \in B(x, \varepsilon) \backslash N\right)
\end{aligned}
$$

Hence we find that $\mathcal{H}_{\kappa}^{s}(\mathcal{F}[f])(x)=$

$$
\bigcap_{\varepsilon>0} \bigcap_{N \in \mathcal{N}} \overline{\operatorname{conv}}\left(\left(d_{x} \Phi^{s}\right)^{-1} d_{z} \Phi^{s} \mathcal{H}_{\kappa}^{s}(f)(z), z \in B(x, \varepsilon) \backslash N\right) .
$$

Let us denote by $\sigma_{\max }\left(\left(d_{x} \Phi^{s}\right)^{-1} d_{z} \Phi^{s}\right)$ the biggest singular value of the linear mapping $\left(d_{x} \Phi^{s}\right)^{-1} d_{z} \Phi^{s}$. The function $\varphi$ : $z \mapsto\left|\sigma_{\max }\left(\left(d_{x} \Phi^{s}\right)^{-1} d_{z} \Phi^{s}\right)-1\right|$ is continuous and therefore 
bounded on $B(x, \varepsilon)$ and moreover vanishes at $z=x$. For all $z \in B(x, \varepsilon)$ we have:

$$
\left\|\left(d_{x} \Phi^{s}\right)^{-1} d_{z} \Phi^{s} \mathcal{H}_{\kappa}^{s}(f)(z)-\mathcal{H}_{\kappa}^{s}(f)(z)\right\| \leq M(\varepsilon),
$$

where $M(\varepsilon)=\sup _{B(x, \varepsilon)} \varphi \operatorname{ess} \sup _{B(x, \varepsilon)}\left\|\mathcal{H}_{\kappa}^{s}(f)\right\|$. The function $M(\varepsilon)$ is continuous at zero and $M(0)=0$. We have proved that $\left(d_{x} \Phi^{s}\right)^{-1} d_{z} \Phi^{s} \mathcal{H}_{\kappa}^{s}(f)(z) \in \mathcal{H}_{\kappa}^{s}(f)(z)+$ $B(0, M(\varepsilon))$. It follows that $\mathcal{H}_{\kappa}^{s}(\mathcal{F}[f])(x)=$

$$
\begin{aligned}
& \bigcap_{\varepsilon>0} \bigcap_{N \in \mathcal{N}} \overline{\operatorname{conv}}\left(\left(d_{x} \Phi^{s}\right)^{-1} d_{z} \Phi^{s} \mathcal{H}_{\kappa}^{s}(f)(z), z \in B(x, \varepsilon) \backslash N\right) \\
= & \bigcap_{\varepsilon>0} \bigcap_{N \in \mathcal{N}} \overline{\operatorname{conv}}\left(\mathcal{H}_{\kappa}^{s}(f)(z)+B(0, M(\varepsilon)), z \in B(x, \varepsilon) \backslash N\right) \\
= & \bigcap_{\varepsilon>0}\left[\bigcap_{N \in \mathcal{N}} \overline{\operatorname{conv}}\left(\mathcal{H}_{\kappa}^{s}(f)(z), z \in B(x, \varepsilon) \backslash N\right)\right) \\
= & \mathcal{F}\left[\mathcal{H}_{\kappa}^{s}(f)\right](x) .
\end{aligned}
$$

The proof of the converse inclusion is similar.

Corollary 2.9: Let $f \in \mathcal{L}_{\text {loc }}^{\infty}\left(\mathbb{R}^{n}, \mathbb{R}^{n}\right)$ be a vector field. Suppose $f$ is $\nu$-homogeneous of degree $\kappa$. Then $\mathcal{F}[f]$ is $\nu$ homogeneous of degree $\kappa$.

Proof: Since $f$ is $\nu$-homogeneous of degree $\kappa$, we have $\mathcal{H}_{\kappa}^{s}(f)=f$. Hence $\mathcal{F}[f]=\mathcal{F}\left[\mathcal{H}_{\kappa}^{s}(f)\right]=\mathcal{H}_{\kappa}^{s}(\mathcal{F}[f])$ by Proposition 2.8 and therefore $\mathcal{F}[f]$ is $\nu$-homogeneous of degree $\kappa$.

The following theorem asserts that a strongly globally asymptotically stable homogeneous differential inclusion admits a homogeneous Lyapunov function. This result is a generalization of the theorem proved for ODE in [10].

Theorem 2.10: [24] Let $F$ be a $\nu$-homogeneous set-valued map of degree $\kappa$, satisfying the SA. Then the following statements are equivalent:

- The origin is (strongly) GAS for the system $\dot{x} \in F(x)$.

- For all $\mu>\max (-\kappa, 0)$, there exists a pair $(V, W)$ of continuous functions, such that:

1) $V \in \mathcal{C}^{\infty}\left(\mathbb{R}^{n}, \mathbb{R}\right), V$ is positive definite and $\nu$ homogeneous of degree $\mu$;

2) $W \in \mathcal{C}^{\infty}\left(\mathbb{R}^{n} \backslash\{0\}, \mathbb{R}\right), W$ is strictly positive outside the origin and $\nu$-homogeneous of degree $\mu+\kappa$;

3) $\max _{v \in F(x)} d_{x} V v \leq-W(x)$ for all $x \neq 0$.

\section{HomogenizATION OF A DIFFERENTIAL INCLUSION}

The following definition extends the notion of local homogeneity to DI.

Definition 3.1: Let $F$ be a set-valued map and $\nu$ be an Euler vector field.

- The set-valued map $H: \mathbb{R}^{n} \rightarrow \mathfrak{H}\left(\mathbb{R}^{n}\right)$ is the $\nu$ homogenization of $F$ at the origin if $H \neq\{0\}$ and if there exists $\kappa \in \mathbb{R}$ such that:

$$
\mathcal{H}_{\kappa}^{s}(F)(x) \underset{s \rightarrow-\infty}{\stackrel{C U C}{\longrightarrow}} H(x) \quad \forall x \in \mathbb{R}^{n} .
$$

- The set-valued map $H: \mathbb{R}^{n} \rightarrow \mathfrak{H}\left(\mathbb{R}^{n}\right)$ is the $\nu$ homogenization of $F$ at infinity if $H \neq\{0\}$ and there exists $\kappa \in \mathbb{R}$ such that:

$$
\mathcal{H}_{\kappa}^{s}(F)(x) \underset{s \rightarrow+\infty}{\stackrel{C U C}{\longrightarrow}} H(x) \quad \forall x \in \mathbb{R}^{n} .
$$

Proposition 3.2: Let $F$ be a set-valued map, $\nu$ be an Euler vector field and $H$ be the $\nu$-homogenization of $F$ at the origin (resp. at infinity). The following properties hold:

1) $H$ is unique;
2) $H$ is $\nu$-homogeneous;

3) If the standard assumptions hold for $F$, they hold for $H$.

Proof: We will only give the proofs in the case of homogenization at the origin, the case of homogenization at infinity being similar.

By uniqueness of the limit, for a given $\kappa \in \mathbb{R}$, the possible limit of $\mathcal{H}_{\kappa}^{s}(F)$ is unique. Assume now that there exists a degree $\mu \neq \kappa$ such that $\mathcal{H}_{\mu}^{s}(F)$ converges to $\tilde{H}$. We will consider two cases.

If $\mu>\kappa$, we have:

$$
\begin{aligned}
\mathfrak{d}\left(\mathcal{H}_{\mu}^{s}(F)(x),\{0\}\right)= & e^{(\kappa-\mu) s} \mathfrak{d}\left(\mathcal{H}_{\kappa}^{s}(F)(x),\{0\}\right) \\
\leq & e^{(\kappa-\mu) s}\left[\mathfrak{d}\left(\mathcal{H}_{\kappa}^{s}(F)(x), H(x)\right)\right. \\
& +\mathfrak{d}(H(x),\{0\})],
\end{aligned}
$$

and therefore for all compact set $X \subset \mathbb{R}^{n}$ :

$$
\begin{gathered}
\sup _{x \in X} \mathfrak{d}\left(\mathcal{H}_{\mu}^{s}(F)(x),\{0\}\right) \leq e^{(\kappa-\mu) s}\left[\sup _{x \in X} \mathfrak{d}\left(\mathcal{H}_{\kappa}^{s}(F)(x), H(x)\right)\right. \\
\left.+\sup _{x \in X} \mathfrak{d}(H(x),\{0\})\right] .
\end{gathered}
$$

Since $\sup _{x \in X} \mathfrak{d}(H(x),\{0\})$ is finite and $\sup _{x \in X} \mathfrak{d}\left(\mathcal{H}_{\kappa}^{s}(F)(x), H(x)\right)$ tends to zero when $s \rightarrow-\infty$, we conclude that $\sup _{x \in X} \mathfrak{d}\left(\mathcal{H}_{\mu}^{s}(F)(x),\{0\}\right) \rightarrow 0$, that is $\tilde{H}=\{0\}$, which is a contradiction.

If $\kappa>\mu$, consider $z \in \mathbb{R}^{n}$ such that $H(z) \neq\{0\}$. The application $X \in \mathfrak{H}\left(\mathbb{R}^{n}\right) \mapsto \sup _{x \in X}\|x\|$ is continuous, hence $\sup _{v \in \mathcal{H}_{\kappa}^{s}(F)(z)}\|v\| \rightarrow \alpha>0$ when $s \rightarrow-\infty$ and therefore $\sup _{v \in \mathcal{H}_{\mu}^{s}(F)(z)}\|v\|=e^{(\mu-\kappa) s} \sup _{v \in \mathcal{H}_{\kappa}^{s}(F)(z)}\|v\| \rightarrow$ $+\infty$ when $s \rightarrow-\infty$, but $\sup _{v \in \mathcal{H}_{\mu}^{s}(F)(z)}\|v\|$ converges to $\sup _{v \in \tilde{H}(z)}\|v\|$ as well and thus $\tilde{H}(z)$ is not bounded, which is a contradiction. This proves the first point.

The homogeneity of $H$ is a consequence of the following computation:

$$
\begin{aligned}
H\left(\Phi^{\sigma}(x)\right)= & \lim _{s \rightarrow-\infty} \mathcal{H}_{\kappa}^{s}(F)\left(\Phi^{\sigma}(x)\right) \\
= & \lim _{s \rightarrow-\infty} e^{-\kappa s}\left(d_{\Phi^{\sigma}(x)} \Phi^{s}\right)^{-1} F\left(\Phi^{s+\sigma(x)}\right) \\
= & \lim _{s \rightarrow-\infty} e^{\kappa \sigma} d_{x} \Phi^{\sigma} e^{-\kappa(s+\sigma)} \times \\
& \left(d_{x} \Phi^{s+\sigma}\right)^{-1} F\left(\Phi^{s+\sigma(x)}(x)\right) \\
= & \lim _{u \rightarrow-\infty} e^{\kappa \sigma} d_{x} \Phi^{\sigma} e^{-\kappa(u)}\left(d_{x} \Phi^{u}\right)^{-1} F\left(\Phi^{u}(x)\right) \\
= & e^{\kappa \sigma} d_{x} \Phi^{\sigma} \lim _{u \rightarrow-\infty} \mathcal{H}_{\kappa}^{u}(F)(x) \\
= & e^{\kappa \sigma} d_{x} \Phi^{\sigma} H(x)
\end{aligned}
$$

Finally, $H(x)$ is a nonempty compact set by construction. It is well known that the convexity is preserved at the limit by the Hausdorff distance, so $H(x)$ is convex. Only the USC remains to prove.

Consider $\mathcal{V}$ an open neighborhood of $H(x)$. We can assume that $\mathcal{V}$ is bounded; if not, we replace it by $\mathcal{V} \cap$ $B(0, r)$ for $r>0$ such that $H(x) \subset B(0, r)$. Denote $\alpha=\inf \{d(h, v), h \in H(x), v \in \partial \mathcal{V}\}>0$. We have $H(x)+$ $B(0, \alpha) \subset \mathcal{V}$. By the uniform convergence, there exists $s$ such that for all $y \in B(x, 1)$, we have $\mathfrak{d}\left(H_{\kappa}^{s}(F)(y), H(y)\right)<$ $\varepsilon / 3$. In particular, $H(y) \subset \mathcal{H}_{\kappa}^{s}(F)(y)+B(0, \varepsilon / 3)$ and $\mathcal{H}_{\kappa}^{s}(F)(x) \subset H(x)+B(0, \varepsilon / 3)$. By USC of $\mathcal{H}_{\kappa}^{s}(F)$, there 
exists a neighborhood of $x, \mathcal{U} \subset B(x, 1)$, such that for all $y \in \mathcal{U}, \mathcal{H}_{\kappa}^{s}(F)(y) \subset \mathcal{H}_{\kappa}^{s}(F)(x)+B(0, \varepsilon / 3)$. Hence, for all $y \in \mathcal{U}, H(y) \subset H_{\kappa}^{s}(F)(y)+B(0, \varepsilon / 3) \subset H_{\kappa}^{s}(F)(x)+$ $B(0,2 \varepsilon / 3) \subset H(x)+B(0, \varepsilon) \subset \mathcal{V}$.

The Definition 3.1 allows us to build a local approximation of a given set-valued map. But we can also apply this procedure to a vector field. Denoting $f$ a locally essentially bounded vector field with a $\nu$-homogenization $h, F$ the regularization of $f$ via the Filippov's procedure and $H$ the $\nu$ homogenization of $F$, then we can naturally wonder whether $\mathcal{F}(h)=H$. The following proposition answers positively to this question.

Proposition 3.3: Consider a locally essentially bounded vector field $f$ with a $\nu$-homogenization $h$ of degree $\kappa$. Then $\mathcal{F}[f]$ admits a $\nu$-homogenization $H$ of degree $\kappa$ and moreover $H=\mathcal{F}[h]$.

Proof: Consider a sequence of locally essentially bounded vector fields $\left(f_{k}\right)$ converging to $f$ uniformly on compact sets. Let us prove that $\mathcal{F}\left(f_{n}\right)$ converges to $\mathcal{F}[f]$ uniformly on compact sets.

For every compact set $Y$, for all $\varepsilon>0$, there exists $N(Y)>0$ such that for all $k \geq N(Y), \sup _{y \in Y} \| f_{n}(y)-$ $f(y) \| \leq \varepsilon$, that is $f_{n}(y) \in f(y)+B(0, \varepsilon)$ and $f(y) \in$ $f_{n}(y)+B(0, \varepsilon)$.

Consider a compact set $X$ and fix $\varepsilon>0$. Denote $Y=X+$ $\bar{B}(0,1)$. For all $x \in X$ and all $\delta<1$ we have $B(x, \delta) \subset Y$. Thus for $n \geq N(Y)$ :

$$
\begin{aligned}
\mathcal{F}\left[f_{n}\right](x) & =\bigcap_{\delta>0} \bigcap_{\mathrm{N} \in \mathcal{N}} \overline{\operatorname{conv}}\left(f_{n}(y), y \in B(x, \delta) \backslash \mathrm{N}\right) \\
& \subset \bigcap_{\delta>0} \bigcap_{\mathrm{N} \in \mathcal{N}} \overline{\operatorname{conv}}(f(y)+B(0, \varepsilon), y \in B(x, \delta) \backslash \mathrm{N}) \\
& \subset \bigcap_{\delta>0} \bigcap_{\mathrm{N} \in \mathcal{N}} \overline{\operatorname{conv}}(f(y), y \in B(x, \delta) \backslash \mathrm{N})+B(0, \varepsilon) \\
& \subset \mathcal{F}[f](x)+B(0, \varepsilon) .
\end{aligned}
$$

The converse inclusion $\mathcal{F}[f](x) \subset \mathcal{F}\left[f_{n}\right](x)+B(0, \varepsilon)$ is obtained similarly. Finally, for $n \geq N(Y)$, for all $x \in$ $X, \mathfrak{d}\left(\mathcal{F}[f](x), \mathcal{F}\left[f_{n}\right](x)\right)<\varepsilon$ and we get the uniform convergence.

Now, by Proposition 2.8, for all $s \in \mathbb{R}$ we have $\mathcal{H}_{\kappa}^{s}(\mathcal{F}[f])=\mathcal{F}\left[\mathcal{H}_{\kappa}^{s}(f)\right]$. Since $\mathcal{H}_{\kappa}^{s}(f)$ is converging uniformly on compact sets to $h, \mathcal{F}\left[\mathcal{H}_{\kappa}^{s}(f)\right]$ converges to $\mathcal{F}[h]$ and hence $\mathcal{H}_{\kappa}^{s}(\mathcal{F}[f])$ converges to $\mathcal{F}[h]$. Since $h$ is $\nu$ homogeneous of degree $\kappa$, so is $\mathcal{F}[h]$ and then by definition $\mathcal{F}[h]$ is the $\nu$-homogenization of $\mathcal{F}[f]$, that is $\mathcal{F}[h]=H$.

Theorem 3.4: Let $F$ be a set-valued map for which the standard assumptions hold and $H$ be its homogenization at the origin. If the origin is a GAS equilibrium of $H$, it is a LAS equilibrium of $F$. If moreover the degree of $H$ is negative, the origin is a locally finite-time stable equilibrium of $F$.

Proof: Let $(V, W)$ be a $\nu$-homogeneous Lyapunov pair for $H$, with $V$ of degree $\mu>\max \{0,-\kappa\}$. Let us denote $\mathbb{S}=$ $\{V=1\}$ and fix $x \in \mathbb{S}$ and $s \in \mathbb{R}$. For the homogenization of $F$ at the origin $H$, we have:

$$
\begin{aligned}
\forall \varepsilon> & 0 \exists g(\varepsilon) \in \mathbb{R}, \forall s \leq g(\varepsilon) \forall x \in \mathbb{S} \\
& \mathfrak{d}\left(\mathcal{H}_{\kappa}^{s}(F)(x), H(x)\right)<\varepsilon .
\end{aligned}
$$

Hence, denoting $a=\inf _{\mathbb{S}} W$ and $b=\sup _{\mathbb{S}}\left\|d_{x} V\right\|$, for all $s \leq g\left(\frac{a}{2 b}\right)$ and all $v \in F\left(\Phi^{s}(x)\right)$, there exists $w \in H(x)$ such that $\left\|e^{-\kappa s}\left(d_{x} \Phi^{s}\right)^{-1} v-w\right\|<\frac{a}{2 b}$. Therefore, for all $s \leq g\left(\frac{a}{2 b}\right)$ and all $v \in F\left(\Phi^{s}(x)\right)$ :

$$
\begin{aligned}
d_{\Phi^{s}(x)} V v= & d_{\Phi^{s}(x)} V\left(v-e^{\kappa s} d_{x} \Phi^{s} w\right)+d_{\Phi^{s}(x)} V\left(e^{\kappa s} d_{x} \Phi^{s} w\right) \\
= & e^{(\kappa+\mu) s}\left[e^{-\mu s} d_{\Phi^{s}(x)} V d_{x} \Phi^{s}\left(e^{-\kappa s}\left(d_{x} \Phi^{s}\right)^{-1} v-w\right)\right. \\
& \left.+e^{-\mu s} d_{\Phi^{s}(x)} V d_{x} \Phi^{s} w\right] \\
= & e^{(\kappa+\mu) s}\left[d_{x} V\left(e^{-\kappa s}\left(d_{x} \Phi^{s}\right)^{-1} v-w\right)+d_{x} V w\right] \\
\leq & e^{(\kappa+\mu) s}\left[b\left\|e^{-\kappa s}\left(d_{x} \Phi^{s}\right)^{-1} v-w\right\|-a\right] \\
\leq & -\frac{a}{2} e^{(\kappa+\mu) s}=-\frac{a}{2} V\left(\Phi^{s}(x)\right)
\end{aligned}
$$

Thus, for all $y \neq 0$ such that $V(y) \leq e^{\kappa g\left(\frac{a}{2}\right)}$, we find that for all $v \in F(y)$ :

$$
d_{y} V v \leq-\frac{a}{2} V(y)^{\frac{\kappa+\mu}{\mu}} .
$$

The relation (7) proves that $V$ is a local Lyapunov function for $F$, and then the origin is a LAS equilibrium of $F$. Moreover, if $\kappa<0$ then $0<\frac{\kappa+\mu}{\mu}<1$. Classical techniques then show that the convergence to the origin is performed in a finite time.

Example 3.5: Consider the following system from [28] (with the particular choice of $\varepsilon=1 / 2$ ):

$$
\left\{\begin{array}{l}
\dot{e}_{1}=e_{2}-k_{1}\left\lfloor e_{1}\right\rceil^{1 / 2}-k_{2} e_{1} \\
\dot{e}_{2}=-k_{3} \operatorname{sign} e_{1}-k_{4} e_{1}
\end{array} .\right.
$$

Taking $\nu=2 x_{1} \frac{\partial}{\partial x_{1}}+x_{2} \frac{\partial}{\partial x_{2}}$, we can compute the $\nu$ homogenization of the system at the origin. A direct computation yields the following $\nu$-homogenization of degree -1 :

$$
\left\{\begin{array}{l}
\dot{e}_{1}=e_{2}-k_{1}\left\lfloor e_{1}\right\rceil^{1 / 2} \\
\dot{e}_{2}=-k_{3} \operatorname{sign} e_{1}
\end{array} .\right.
$$

The origin is known to be globally finite-time stable for this system, and we conclude by the Theorem 3.4 that the origin is a locally finite-time stable equilibrium of the initial system.

\section{ROBUSTNESS OF HOMOGENEOUS AND HOMOGENIZABLE SYSTEMS}

In this section we consider a measurable set-valued map $F: \mathbb{R}^{n} \times \mathbb{R}^{m} \rightrightarrows \mathbb{R}^{n}$. We denote $F_{d}(x)=F(x, d)$. We will be interested in proving robustness properties of the system defined by:

$$
\dot{x} \in F(x, d), \quad x \in \mathbb{R}^{n}, \quad d \in \mathcal{L}_{\text {loc }}^{\infty}\left(\mathbb{R}, \mathbb{R}^{m}\right) .
$$

\section{A. ISS definitions and properties}

In this work we will be interested in the following stability properties [7], [29].

Definition 4.1: The system (8) is called input-to-state practically stable (ISpS), if for any input $d \in \mathcal{L}_{\text {loc }}^{\infty}\left(\mathbb{R}, \mathbb{R}^{m}\right)$ and any $x_{0} \in \mathbb{R}^{n}$ there are some functions $\beta \in \mathcal{K} \mathcal{L}, \gamma \in \mathcal{K}$ and $c \geq 0$ such that for any solution $x$ of (8):

$$
\|x(t)\| \leq \beta\left(\left\|x_{0}\right\|, t\right)+\gamma\left(\|d\|_{[0, t]}\right)+c \quad \forall t \geq 0 .
$$

The function $\gamma$ is called nonlinear asymptotic gain. The system is called ISS if $c=0$. 
These properties have the following Lyapunov function characterizations.

Definition 4.2: A smooth function $V: \mathbb{R}^{n} \rightarrow \mathbb{R}_{+}$is called ISpS Lyapunov function for the system (8) if for all $x \in \mathbb{R}^{n}$, $d \in \mathbb{R}^{m}$ and some $r \geq 0, \alpha_{1}, \alpha_{2}, \alpha_{3} \in \mathcal{K}_{\infty}$ and $\theta \in \mathcal{K}$ :

$$
\begin{aligned}
\alpha_{1}(\|x\|) & \leq V(x) \leq \alpha_{2}(\|x\|), \\
\sup _{v \in F(x, d)} d_{x} V v & \leq r+\theta(\|d\|)-\alpha_{3}(\|x\|) .
\end{aligned}
$$

Such a function $V$ is called ISS Lyapunov function if $r=0$. Note that an ISS Lyapunov function can also satisfy the following equivalent condition for some $\alpha_{4}, \chi \in \mathcal{K}_{\infty}$ and $\rho \geq 0$ :

$$
\|x\|>\chi(\|d\|)+\rho \Rightarrow \sup _{v \in F(x, d)} d_{x} V v \leq-\alpha_{4}(\|x\|) .
$$

Proposition 4.3: If there exists an ISpS (resp. ISS) Lyapunov function for the system (8), then the system is ISpS (resp. ISS).

\section{B. ISS of homogeneous differential inclusions}

In the following results, we will need some assumptions on $F$.

Assumption 1: For all $d \in \mathbb{R}^{m}$ the set-valued map $F_{d}$ verifies the SA.

This assumption ensures that solutions of the system (8) exist.

Assumption 2: There exists a $\nu$-homogeneous set-valued map $H$ of degree $\kappa$ verifying the SA such that

A. the origin is a GAS equilibrium of $H$. We denote $(V, W)$ a $\nu$-homogeneous Lyapunov pair for $H$ given by Theorem 2.10 .

B. for all $\varepsilon>0$ and for all $D \geq 0$ there exists $\eta>0$ such that for all $s \geq \eta$, for all $x \in \mathbb{S}=\{V=1\}$ and for all $\|d\| \leq D$, we have $\mathcal{H}_{\kappa}^{s}\left(F_{d}\right)(x) \subset H(x)+B(0, \varepsilon)$.

Following the notations used in Section III, we denote $a=$ $\inf _{\mathbb{S}} W$ and $b=\sup _{\mathbb{S}}\left\|d_{x} V\right\|$. We also denote

$h(D)=\inf \left\{\eta \in \mathbb{R}: \forall s \geq \eta \mathcal{H}_{\kappa}^{s}\left(F_{d}\right)(x) \subset H(x)+B\left(0, \frac{a}{2 b}\right)\right\}$.

By Assumption $2 \mathrm{~B}, h(D)<+\infty$. We allow $h(D)=-\infty$, denote $\ell=\lim _{D \rightarrow 0^{+}} h(D)$.

Theorem 4.4: Under Assumptions 1 and 2, the system (8) is:

ISS if $\ell=-\infty$,

ISpS if $\ell \neq-\infty$.

Remark 4.5: The following hint for a selection of $H$ can be proposed. When $F_{0}$ is $\nu$-homogeneous of degree $\mu$, Assumption 2B gives, for $x \in \mathbb{S}$ : $\lim _{s \rightarrow+\infty} e^{(\mu-\kappa) s} F_{0}(x) \subset$ $H(x)$. If $\mu>\kappa, e^{(\mu-\kappa) s} F_{0}(x)$ diverges when $s \rightarrow+\infty$, and if $\mu<\kappa$, we get that $0 \in H(x)$, which is a contradiction to the global asymptotic stability of $H$ (Assumption 2A). Thus $\mu=\kappa$ and $F_{0} \subset H$. Similarly, if $F_{0}$ admits a $\nu$ homogeneization $H_{0}$ of degree $\mu$, we find that $\mu=\kappa$ and $H_{0} \subset H$. This remark gives us a candidate for $H$ in some situations and it will be used in Theorem 4.10.

To prove the Theorem 4.4, we need some technical lemmas, which proofs are omitted due to space limitations.
Lemma 4.6: Let $\sigma: \mathbb{R}_{+} \rightarrow \mathbb{R}$ be an increasing function such that $\lim _{x \rightarrow 0^{+}} \sigma(x)=0$. Then there exists a class $\mathcal{K}$ function $\bar{\sigma}$ such that for all $x \in \mathbb{R}_{+}, \sigma(x) \leq \bar{\sigma}(x)$.

Lemma 4.7: Let $V: \mathbb{R}^{n} \rightarrow \mathbb{R}$ be a positive definite $\nu$ homogeneous function of degree $\kappa>0$. There exist $\sigma_{-}$and $\sigma_{+}$two functions of class $\mathcal{K}$ such that for all $x \in \mathbb{R}^{n}$ :

$$
\sigma_{-}(\|x\|) \leq V(x) \leq \sigma_{+}(\|x\|) .
$$

The proof of Theorem 4.4 is omitted due to space limitations. It is based on the following inequality and the successive use of the beforehand Lemmas:

$$
\sup _{v \in F_{d}(y)} d_{y} V v \leq-\frac{a}{2} V(y)^{\frac{\kappa+\mu}{\mu}} .
$$

Inequality 9 gives one straightforward Corollary.

Corollary 4.8: Under Assumptions 1 and 2, if $\kappa>0$, then the system (8) is ISS. If moreover we denote $\gamma$ the asymptotic gain, then any trajectory of the system converges to the ball of radius $\gamma\left(\|d\|_{\infty}\right)$ in a uniform finite-time.

The following Corollary of Theorem 4.4 shows how to use it when dealing with more concrete systems.

Corollary 4.9: Consider the system $\dot{x} \in F_{0}(x)+B(0,|d|)$. Assume that:

1) $F_{0}$ verifies the $\mathrm{SA}$;

2) the origin is a GAS equilibrium of $F_{0}$;

3 ) there exists a linear Euler vector field $\nu(x)=A x$ such that $F_{0}$ is $\nu$-homogeneous of degree $\kappa$;

4) if $\rho$ denotes the smallest real part of the eigenvalues of $A, \kappa+\rho>0$.

Then the system $\dot{x} \in F_{0}(x)+B(0,|d|)$ is ISS.

Proof: Assumption 1 and $2 \mathrm{~A}$ are clearly verified, taking $H=F_{0}$. Noting that $\mathcal{H}_{\kappa}^{s}\left(F_{d}\right)(x)=F_{0}(x)+$ $\exp ((-\kappa I-A) s) B(0,|d|)$, we see that Assumption $2 \mathrm{~B}$ holds because the matrix $-\kappa I-A$ is Hurwitz $(\rho+\kappa>$ $0)$. Finally, $\lim _{D \rightarrow 0^{+}} h(D)=-\infty$ follows from classical matrices considerations.

Theorem 4.10: Consider a set-valued map $F: \mathbb{R}^{n} \times$ $\mathbb{R}^{m} \rightrightarrows \mathbb{R}^{n}$ verifying Assumption 1. Assume moreover that the following hypothesis hold:

1) The origin is a GAS equilibrium of $F_{0}$. We denote $(V, W)$ a $\nu$-homogeneous Lyapunov pair for $F_{0}$ given by Theorem 2.10 .

2) There exists an Euler vector field $\tilde{\nu}$ on $\mathbb{R}^{m}$, which flow is denoted $\tilde{\Phi}$, such that:

$$
F\left(\Phi^{s}(x), \tilde{\Phi}^{s}(d)\right)=e^{\kappa s} d_{x} \Phi^{s} F(x, d) .
$$

3) There exists a function $\sigma \in \mathcal{K}$ such that for all $x \in \mathbb{S}=$ $\{V=1\}$ we have $F_{d}(x) \subset F_{0}(x)+B(0, \sigma(\|d\|))$.

Then the system (8) is ISS.

Proof: Let us show first that Assumption 2 holds for $H=F_{0}$. The point $2 \mathrm{~A}$ is given by the point 1 of the hypothesis of this theorem.

By the point 2 of the hypothesis, $\mathcal{H}_{\kappa}^{s}\left(F_{d}\right)(x)=$ $F_{\tilde{\Phi}^{-s}(d)}(x)$. Consider $N$ a $\tilde{\nu}$-homogeneous norm on $\mathbb{R}^{m}$ and denote $\eta(\varepsilon, D)=\ln \left(\frac{\sigma_{+}(D)}{\sigma_{-} \circ \sigma^{-1}(\varepsilon)}\right)$, where the functions $\sigma_{-}$and $\sigma_{+}$are given by Lemma 4.7 with respect to the function $N$. Consider $s \geq \eta(\varepsilon, D)$. Then 
$e^{-s} \sigma_{+}(D) \leq \sigma_{-} \circ \sigma^{-1}(\varepsilon)$ and for all $\|d\| \leq D$ we have $\left\|\tilde{\Phi}^{-s}(\bar{d})\right\| \leq \sigma_{-}^{-1}\left(N\left(\tilde{\Phi}^{-s}(d)\right)\right) \leq \sigma_{-}^{-1}\left(e^{-s} N(d)\right) \leq$ $\sigma_{-}^{-1}\left(e^{-s} \sigma_{+}(\|d\|)\right) \leq \sigma^{-1}(\varepsilon)$. Hence for all $s \geq \eta(\varepsilon, D)$, for all $x \in \mathbb{S}$ and all $\|d\| \leq D$ we have $\mathcal{H}_{\kappa}^{s}\left(F_{d}\right)(x)=$ $F_{\tilde{\Phi}^{-s}(d)}(x) \subset F_{0}(x)+B(0, \varepsilon)$ by hypothesis 3 . Therefore Assumption 2 holds for $H=F_{0}$. Since $h(D) \leq \eta\left(\frac{a}{2 b}, D\right)$ and $\lim _{D \rightarrow 0^{+}} \eta\left(\frac{a}{2 b}, D\right)=-\infty$, we conclude by Theorem 4.4.

Example 4.11: Consider the following disturbed system:

$$
\left\{\begin{array}{l}
\dot{x}_{1}=x_{2}+d \\
\dot{x}_{2}=-k_{1} \operatorname{sign}\left(x_{1}\right)-k_{2} \operatorname{sign}\left(x_{2}\right)
\end{array}\right.
$$

where $k_{1}>k_{2}>0$ are fixed gains. When $d=0$, it is wellknown that the system is $\nu$-homogeneous of degree $\kappa=-1$ with $\nu=2 x_{1} \frac{\partial}{\partial x_{1}}+x_{2} \frac{\partial}{\partial x_{2}}$ and GAS, that is hypothesis 1 holds. Taking $\tilde{\nu}=d \frac{\partial}{\partial d}$, we see that hypothesis 2 holds. Finally the hypothesis 3 also holds with $\sigma(D)=D$ and the system is ISS by Theorem 4.10 .

Corollary 4.12: Let $f: \mathbb{R}^{n} \times \mathbb{R}^{m} \rightarrow \mathbb{R}^{n}$ be a continuous vector field. Assume that there exists an Euler vector field $\tilde{\nu}$ on $\mathbb{R}^{m}$, which flow is denoted $\tilde{\Phi}$, such that:

$$
f\left(\Phi^{s}(x), \tilde{\Phi}^{s}(d)\right)=e^{\kappa s} d_{x} \Phi^{s} f(x, d),
$$

and assume moreover that the origin is a GAS equilibrium of $f_{0}$. Then the system $\dot{x}=f(x, d)$ is ISS.

Proof: We take $F_{d}(x)=\left\{f_{d}(x)\right\}$. The hypothesis 1 and 2 of Theorem 4.10 are clearly satisfied. The continuity of $f$ and the compactness of $\mathbb{S}$ give that the function $\sigma(d)=$ $\sup _{x \in \mathbb{S},\|d\| \leq D}\left\|f_{d}(x)-f_{0}(x)\right\|$ belongs to class $\mathcal{K}$, which gives in turn hypothesis 3 and concludes the proof.

\section{CONCLUSION}

In this paper, we achieved two objectives. First, we introduced local homogeneity for DI. We proved that this notion is consistent with the Filippov's procedure and that the local stability is inherited by a system which has a GAS homogenization. Second, we applied homogeneity and local homogeneity techniques to prove ISS and ISpS properties of systems defined by DI. All these results were presented using geometric homogeneity.

In the future, we plan to use these results and techniques for designing SMC and getting a good understanding of the associated robustness properties of such systems. In particular, controlling the asymptotic gain could give a way of reducing the chattering effect.

\section{REFERENCES}

[1] J. Doyle, B. Francis, and A. Tannenbaum, Feedback Control Systems. Mac Millan Publishing Co, 1992.

[2] D. J. Hill and P. J. Moylan, "Dissipative dynamical systems: basic input-output and state properties," J. Franklin Inst., vol. 309, no. 5, pp. 327-357, 1980.

[3] A. van der Schaft, $L_{2}$-gain and passivity techniques in nonlinear control, ser. Lecture Notes in Control and Information Sciences. London: Springer-Verlag London Ltd., 1996, vol. 218.

[4] E. D. Sontag, "The ISS philosophy as a unifying framework for stability-like behavior," in Nonlinear control in the year 2000, Vol. 2 (Paris), ser. Lecture Notes in Control and Inform. Sci. London: Springer, 2001, vol. 259, pp. 443-467.
[5] M. Vidyasagar, Input-output analysis of large-scale interconnected systems, ser. Lecture Notes in Control and Information Sciences. Berlin: Springer-Verlag, 1981, vol. 29, decomposition, well-posedness and stability.

[6] J. C. Willems, "Dissipative dynamical systems. I. General theory," Arch. Rational Mech. Anal., vol. 45, pp. 321-351, 1972.

[7] E. D. Sontag, "Smooth stabilization implies coprime factorization," IEEE Trans. Automat. Control, vol. 34, no. 4, pp. 435-443, 1989.

[8] V. Zubov, "On ordinary differential equations with generalized homogeneous right-hand sides," Izvestiya vuzov, Matematika, vol. 1, no. 2, pp. 80-88, 1958.

[9] M. Kawski, "Nilpotent lie algebras of vectorfields," J. reine angew. Math, vol. 388, pp. 1-17, 1988.

[10] L. Rosier, "Homogeneous Lyapunov function for homogeneous continuous vector field," Systems \& Control Letters, vol. 19, pp. 467-473, 1992.

[11] H. Hermes, "Homogeneous feedback controls for homogeneous systems," Systems \& Control Letters, vol. 24, pp. 7-11, 1995.

[12] L. Grüne, "Homogeneous state feedback stabilization of homogeneous systems," SIAM J. Control Optimization, vol. 38, no. 4, pp. 1288-1314, 2000.

[13] S. P. Bhat and D. S. Bernstein, "Geometric homogeneity with applications to finite-time stability," Mathematics of Control, Signals and Systems, vol. 17, pp. 101-127, 2005.

[14] V. Bokharaie, O. Mason, and M. Verwoerd, "D-stability and delayindependent stability of homogeneous cooperative systems," Automatic Control, IEEE Transactions on, vol. 55, no. 12, pp. 2882-2885, 2010.

[15] A. Aleksandrov, A. Kosov, and A. Platonov, "On the asymptotic stability of switched homogeneous systems," Systems \& Control Letters, vol. 61 , no. 1, pp. 127-133, 2012.

[16] V. Andrieu, L. Praly, and A. Astolfi, "Homogeneous Approximation, Recursive Observer Design, and Output Feedback," SIAM J. Control Optimization, vol. 47, no. 4, pp. 1814-1850, 2008.

[17] D. Efimov and W. Perruquetti, "Oscillations conditions in homogenous systems," in Proc. IFAC NOLCOS Symp., 2010, pp. 1379-1384.

[18] Y. Hong, " $\mathrm{H}_{\infty}$ control, stabilization, and input-output stability of nonlinear systems with homogeneous properties," Automatica, vol. 37, no. 7, pp. 819-829, 2001.

[19] E. Ryan, "Universal stabilization of a class of nonlinear systems with homogeneous vector fields," Systems \& Control Letters, vol. 26, pp. 177-184, 1995.

[20] E. Bernuau, D. Efimov, and W. Perruquetti, "On a coordinate-free transition from global to local homogeneity," Submitted in Systems and Control Letters, 2013.

[21] A. Filippov, Differential Equations with Discontinuous Righthand Sides, F. Arscott, Ed. Kluwer Academic, 1988.

[22] A. Levant, "Homogeneity approach to high-order sliding mode design," Automatica, vol. 41, no. 5, pp. 823-830, 2005.

[23] Y. Orlov, "Finite time stability and robust control synthesis of uncertain switched systems," SIAM J. Control Optim., vol. 43, no. 4, pp. 12531271,2005

[24] E. Bernuau, A. Polyakov, D. Efimov, and W. Perruquetti, "On extension of homogeneity notion for differential inclusions," in Proc. of ECC 2013, 2013

[25] —_ "Robustness of finite-time stability property for sliding modes," in Proc. of 5th IFAC Symposium on System Structure and Control, 2013.

[26] J.-P. Aubin and A. Cellina, Differential Inclusions, ser. Grundlehren der Math. Wiisenschaften 264. Springer-Verlag, 1984.

[27] M. Kawski, "Families of dilations and asymptotic stability," in Analysis of controlled dynamical systems (Lyon, 1990), ser. Progr. Systems Control Theory. Boston, MA: Birkhäuser Boston, 1991, vol. 8, pp. 285-294.

[28] Y. Orlov, Y. Aoustin, and C. Chevallereau, "Finite time stabilization of a perturbed double integrator - part i: Continuous sliding mode-based output feedback synthesis," IEEE Transactions on Automatic Control, vol. 56, no. 3, pp. 614-618, 2011.

[29] E. D. Sontag and Y. Wang, "On characterizations of the input-to-state stability property," Systems Control Lett., vol. 24, no. 5, pp. 351-359, 1995. 